\title{
Systemic Kikuchi-Fujimoto disease bordering lupus lymphadenitis: A fresh look?
}

\author{
Aram Behdadnia ${ }^{1}$, Seyyed Farshad Allameh ${ }^{2}$, Mehrnaz Asadi Gharabaghi ${ }^{3}$, Seyed Reza Najafizadeh ${ }^{1}$, \\ Ahmad Tahamoli Roudsari ${ }^{1}$, Alireza Ghajar ${ }^{1,4}$, Morsaleh Ganji ${ }^{1,4}$, Mohsen Afarideh ${ }^{1,4}$, \\ ${ }^{1}$ Lupus and Rheumatology department, Vali-Asr Hospital, School of Medicine, Tehran University of Medical Sciences, Tehran, Iran; \\ ${ }^{2}$ Department of General Internal Medicine, Imam Khomeini Hospital, School of Medicine, Tehran University of Medical Sciences, \\ Tehran, Iran: \\ ${ }^{3}$ Department of Pulmonary Medicine, School of Medicine, Tehran University of Medical Sciences, Tehran, Iran, \\ ${ }^{4}$ Endocrinology and Metabolism Research Center (EMRC), Vali-Asr Hospital, School of Medicine, Tehran University of Medical \\ Sciences, Tehran, Iran.
}

\begin{abstract}
Summary A 31-year old woman with persistent fever for 6 weeks and unresponsive to antibiotic therapy came for rheumatologic investigation. After computed tomography (CT) studies of her neck, thorax and abdomen revealed bilateral cervical, axillary and retroperitoneal lymph node enlargements, histopathologic evaluation of the resected nodes showed features of histiocytic necrotizing lymphadenopathy suggestive of Kikuchi-Fujimoto's lymphadenopathy. KikuchiFujimoto Disease (KFD) involving the retroperitoneal nodes is extremely unusual and even more challenging to diagnose when there are no early signs of extranodal involvement or abdominopelvic pain. We present a case of systemic KFD involving the cervical, axillary and retroperitoneal lymph nodes and emphasize the clinical interest to properly differentiate between the benign condition of KFD that requires no more than minimal to low dosage steroid therapy and the potentially life-threatening lupus lymphadenitis that mandates intensive immunosuppressive treatment.
\end{abstract}

Keywords: Kikuchi-Fujimoto disease, necrotizing lymphadenitis, systemic lupus erythematosus, lupus lymphadenitis

\section{Introduction}

Kikuchi-Fujimoto's necrotizing lymphadenitis is a predominantly benign disease characterized by regional lymphadenopathy, low-grade fever and night sweats often involving the cervical lymph nodes in young people with less frequent involvement of lymph nodes elsewhere (1). From 1972 with the first reports of Kikuchi-Fujimoto disease (KFD) in Japan (2), the knowledge of KFD has been confined to case reports and series. This lack of evidence surrounding the pathophysiology of KFD has caused two major questions: $i$ ) does KFD belong

Released online in J-STAGE as advance publication October 24, 2016.

*Address correspondence to:

Dr. Mohsen Afarideh, Lupus and Rheumatology department, Vali-Asr Hospital, Tehran University of Medical Sciences, P.O. Box 13145-784, Tehran, Iran.

E-mail: mhafarideh@gmail.com to an independent category of autoimmune diseases or ii) does KFD develop as a bystander autoimmunelike phenomenon in many autoimmune diseases (e.g. systemic lupus erythematosus, SLE)? Credentials of the earlier suggestion have been put into question by two major findings: $i$ ) the association of SLE with KFD is well documented in the literature and the importance of lymph node biopsy is also well established; $i$ ) in addition to SLE, KFD occurs in many other autoimmune diseases $(3,4)$. Here we delineate a 31 -year old woman with a 5-year history of SLE. In this case, two points may lead us into the diagnosis of KFD: first, the distinct clinicopathologic features of KFD as reported from the biopsy of resected nodes; and second, a demographic profile of the patient that fits well with the epidemiological background of KFD.

\section{Case Report}

A 31 year old G3P1 Ab2 (7 months, 4 months) woman 
was admitted into our unit (Lupus and Rheumatology department, Vali-Asr Hospital) due to persisting complaints of 6-week unremitting low-grade fever and chills. The fever was persistent in nature with no defining pattern and aggravating factors, however it was relieved to some extent with acetaminophen intake and was associated with, nausea, vomiting, malaise, arthralgia, nocturnal diaphoresis and a weight loss of $15 \mathrm{lb}$. during the past 40 days. From the age of 19 with a diagnosis of rheumatoid arthritis she has been on chloroquine $5 \mathrm{mg} / \mathrm{kg}$ /day and 5 years ago, she was diagnosed with SLE based on the findings of the American College of Rheumatology (ACR) criteria: presence of photosensitivity, malar rash, large symmetric joint arthritis involvement and positive antinuclear antibody (ANA). She denied chest pain, shortness of breath, dizziness, diarrhea, urinary symptoms, vaginal discharge, rash, headache, myalgia or rigor. She denied sick contacts, recent travel, exposure to pets/animals, bug bites, new medications, and herbal supplements. Her drug history included prednisone $5 \mathrm{mg}$ /day, hydroxychloroquine 200-400 $\mathrm{mg}$ /day and folinic acid. Prior to admission, the patient was evaluated at a community urgent care center for possible infectious etiologies and was started on IV broad-spectrum antibiotic therapy; however, her condition remained unresponsive to this treatment and she was subsequently presented to our department. Her past medical was remarkable for minor $\beta$-thalassemia and cataract surgery. On physical examination, the patient was pale, she was febrile (101.48 F), tachycardic at 100 , and had a blood pressure of 100/60. Physical exam showed diffuse, rubbery and slightly tender enlargement of bilateral posterior cervical lymph nodes and bilateral axillary lymph nodes with the largest node measuring to $1.5 \mathrm{~cm}$. Enlarged lymph nodes were noted to be firm, discrete and mobile. There was no joint tenderness, warmness, erythema, swelling, limited range of motion or stiffness. Examination of the gastrointestinal, respiratory, cardiovascular and nervous systems was normal with no hepatosplenomegaly.

\subsection{Investigations}

Lab Investigations revealed hemoglobin $9.3 \mathrm{~g} / \mathrm{dL}$, WBC count 11,960 cells/mm (neutrophils-51\% , lymphocytes-36\% with no atypical lymphocytes) with platelet count of 276,000 cells/cu.mm, erythrocyte sedimentation rate $(\mathrm{ESR})-30 \mathrm{~mm} / \mathrm{hr}$ and C-reactive protein (CRP) $-6.7 \mathrm{mg} / \mathrm{dL}$; alanine aminotransferase (ALAT) $15 \mathrm{IU} / \mathrm{L}$ (normal up to 31); aspartate aminotransferase (ASAT) 24 IU/L (normal up to 31); alkaline phosphatase (ALP) 94 IU/L (64-306); serum lactate dehydrogenase (LDH) 493 U/L (normal 225500); serum Iron $57 \mu \mathrm{g} / \mathrm{dL}$ (37-158), ferritin $110 \mathrm{ng} /$ $\mathrm{mL}(10-126 \mathrm{ng} / \mathrm{mL})$, total iron-binding capacity (TIBC) $289 \mu \mathrm{g} / \mathrm{dL}$ (210-440); serum 25-hydroxyvitamin D level 30.56 (desirable $>30$ ); ANA positive with a titer of 1:80; complement $\mathrm{C} 3$ and $\mathrm{C} 4$ were decreased [C3 32.2 (reference: 90-180), C4 3.1 (reference: 10-40)] ; low $\mathrm{CH} 50$ at 30.5; the anti-double stranded DNA (antidsDNA) positive at $141.2 \mathrm{IU} / \mathrm{mL}$. Additional tests including for HIV (EL1SA antibody), tuberculosis (PPD and Chest X-ray, a positive PPD skin test was defined as $\geq 10 \mathrm{~mm}$ intradermal induration), brucellosis (Wright, Coombs W.), typhoid (Widal TO and TH serology) and repeated blood cultures were negative. Urinalysis was normal except for a sterile pyuria (U/A: WBC, 16-18; RBC, 1-2; few epithelial cells; specific gravity, 1,005; negative nitrate; negative proteinuria) with no evidence of infection (Zero colony count); renal parameters were within normal ranges as were her thyroid function tests.

Double-contrast Spiral computed tomography (CT) of the abdominopelvic region revealed enlarged retroperitoneal lymph nodes in lateral aspect of the aorta at the level of the renal hilum, with no evidence of hepato-splenomegaly, ascites and bowel thickening. IV contrast CT study of the neck and thorax was noticeable for cervical lymphadenopathy located bilaterally in levels 1, 2 and 3 and left-sided in levels 4 and 5 and bilateral axillary enlarged nodes, with no mediastinal mass lesion, nodule, effusion, thickening or lymphadenopathy being detected. Maximum short axis diameters for enlarged lymph nodes were $8 \mathrm{~mm}$ and 15 $\mathrm{mm}$ for cervical and axillary lymph nodes, respectively.

\subsection{Outcome, treatment and follow-up}

With the initial diagnosis of lupus flare-up, the patient had dramatic clinical improvement to an increased daily dose of prednisolone to $10 \mathrm{mg}$ and the constellation of her symptoms concerning cervical and axillary lymphadenopathies promptly resolved. Following the core needle biopsy of the cervical enlarged lymph nodes, the patient was discharged from the hospital. In the ensuing pathology report, histology of the resected nodes indicated the nodal architecture was preserved, as highlighted by CD20 stain of follicular germinal centers which were bcl 2 negative. The parenchyma contained areas of karyorrhectic necrosis, with no neutrophilic reaction surrounded by plasmacytoid monocytes and lymphoid cells. The biopsy report confirmed these findings were consistent with the diagnosis of histiocytic necrotizing lymphadenitis (Kikuchi Fujimoto disease). Three months later, the patient emerged in our unit complaining of daily spells of abdominal pain and nausea/vomiting. In that time, she was diagnosed with lupus necrotizing pancreatitis and started on pulsed methylprednisolone and endoxan. Induction treatment by pulsed methyl prednisolone was received in daily doses of $1 \mathrm{~g}$ IV for 3 days, followed by monthly injections of $1 \mathrm{~g}$ IV endoxan for 7 months. Oral prednisone was initiated on the fourth day of treatment, at $1 \mathrm{mg} / \mathrm{kg}$ per day, for no more than 3 
months and was tapered off in 4 to 6 months thereafter. She was in total remission with cellcept which was started on the seventh month after the manifestation of necrotizing pancreatitis, at a daily dosage of $2 \mathrm{mg} / \mathrm{kg}$ (1 $\mathrm{mg} / \mathrm{kg} \mathrm{BID)}$, and continued for 1.5 years.

\section{Discussion}

KFD is characterized as a rare, benign and selfremitting condition that is likely to be caused by a strong immunological response to a viral infection (5). However, the evidence linking KFD to a specific viral infection such as Epstein-Barr virus (EBV) and Herpes virus 6 are scarce $(6,7)$ and numerous other inciting infectious and autoimmune triggers have been described, including the role of SLE in the pathogenesis of KFD. Affected women of Asian background in their early twenties to their mid-thirties typify the classic picture of KFD that is most commonly characterized by acute/ subacute febrile illness associated with lymph node enlargement of mainly posterior cervical lymph nodes (2). Our patient manifested bilateral cervical and axillary lymphadenopathy as well as engorged retroperitoneal lymph nodes. Generalized lymphadenopathy can present occasionally, but involvement of mediastinal or retroperitoneal nodes by KFD is extremely uncommon (8). Other accompanying symptoms in KFD include malaise, anorexia, myalgias and arthralgias (2).

Though the diagnosis of KFD is made prior to, simultaneous to or after the diagnosis of SLE (9), review of the literature in published cases from 1991 onwards indicates that SLE predominantly predates or concurs with KFD. However, it is not clear whether those cases were genuine KFD or were indeed lupus lymphadenitis mimicking KFD because lupus lymphadenitis is clinically indistinguishable from KFD in the absence of hematoxylin bodies and abundant plasma cells. For this reason, the important diagnostic challenge is to differentiate KFD from the more common entity of lupus lymphadenitis. Considering the potentially lifethreatening nature of lupus lymphadenitis versus the self-limiting character of KFD, lupus lymphadenitis should always be suspected in patients with SLE who present with necrotizing lymphadenitis. To this aim, careful examination of lymph node histopathology in correlation with clinical features is the most reliable way to differentiate KFD from lupus lymphadenitis and other serious entities that it may mimic, such as fulminant EBV-infection and T-cell lymphoma. For instance, The CD20 and CD3 staining results in our case do not support a diagnosis of B- or T-cell lymphoma.

Depending on the stage of disease, KFD is demonstrated by cortical and paracortical necrotizing nodules, apoptotic debris, proliferation of CD68 histiocytes and plasmacytoid monocytes, immunoblasts, abundant CD8 T-cells, and an absence/paucity of neutrophils $(7,10)$. In contrast, lupus lymphadenitis is diagnosed in patients who meet the validated revised ACR criteria for SLE together with typical biopsy findings of necrotic and thrombosed blood vessels, presence of necrotizing neutrophilic infiltrates as is seen in drug-induced lymphadenopathy and the pathognomonic feature, hematoxylin bodies (7). However, it is suggested that there may be a significant overlap between pathomorphological features of KFD and lupus lymphadenitis. Therefore, it is imperative for pathologists to be aware of the possibility that lupus lymphadenitis may mimic KFD even on histopathology, and pathologists should inform the clinician of this possibility. The pathologist should also become alert to the possible diagnosis of lupus lymphadenitis if extensive necrosis is seen (as in our case; Table 1). It is commonly accepted that hematoxylin bodies which resemble condensed complexes of DNA and antidsDNA-antibodies discriminate between KFD and SLE as ANA, antiphospholipid antibody (APLA), Anti-dsDNA, rheumatoid factor (RF) are all usually negative in KFD; although positive ANA ( like in our case ) has also been reported in patients with KFD (11). Nevertheless, a high level of Anti-dsDNA in our case favors the diagnosis of lupus lymphadenitis. Meanwhile, presence of abundant cytotoxic T-cells on histopathology (12), which was a finding in our case, is consistent with diagnosis of KFD. However, it has also been suggested that patients with KFD tend to have higher incidence rates of cutaneous manifestations (13) which was not present in our patient.

Despite the characteristic histopathologic diversity, clinical features of SLE and KFD can be very similar; fever, lymphadenopathy, fatigue, and joint pain can be seen in both diseases. Hence, there is an ongoing debate on whether KFD is indeed an atypical manifestation of lupus lymphadenitis. Cramer et al. in their report suggested that KFD may be a histopathologic alternative form of lupus lymphadenitis representing a 'forme fruste' rather than being an independent disease entity (14). Reported estimates from lupus lymphadenitis range between $12 \%$ to $58 \%$ of patients with SLE (15), far more common than that of KFD. Perhaps, one of the reasons for such rarity of reported cases with KFD is that lymph node biopsy is seldom performed in patients with SLE to establish the diagnosis of either KFD or lupus lymphadenitis. We believe the concurrent presence of positive ANA/Anti-ds DNA/ low C3, C4, which support a diagnosis of SLE and pathological evidence suggestive of KFD in our case may imply that lupus lymphadenitis and KFD could in fact belong to the same entity.

The course of KFD is benign and self-limiting in the overwhelming majority of patients, most often resolving within several weeks to 6 months after the initial diagnosis $(15,16)$. However and based on the natural history, a thorough prediction of the severity of outcomes can be challenging in some cases. The majority of patients with KFD have a normal complete 
Table 1. Anti-HBV response of TCM and related active compounds in clinical trials

\begin{tabular}{|c|c|}
\hline Items & Message \\
\hline 1 & $\begin{array}{l}\text { The association between SLE and KFD is well documented in the literature and the importance of lymph node biopsy in guiding the } \\
\text { definitive diagnosis is also well established. }\end{array}$ \\
\hline 2 & $\begin{array}{l}\text { Characteristic morphology and histopathology of the resected lymph nodes should thoroughly distinguish KFD (particularly the type } \\
\text { with necrotizing pathology) from lupus lymphadenitis since these two entities have different management protocols. }\end{array}$ \\
\hline 3 & $\begin{array}{l}\text { Regular follow-up visits are recommended in more severe and systemic manifestations of KFD from three critical aspects: First, } \\
\text { to preclude relapses of coexisting SLE in those already diagnosed with SLE; second, to follow the recurrences of KFD which are } \\
\text { generally not worrisome; and third to watch for possible development of lupus lymphadenitis which is of significant clinical urgency } \\
\text { for careful management. }\end{array}$ \\
\hline 4 & $\begin{array}{l}\text { Laboratory measures of ANA, APLA, Anti-dsDNA and RF may not be accurate and reliable indicators to differentiate correctly } \\
\text { between lupus lymphadenitis and KFD. Instead, histopathologic features such as abundant specimen cytotoxic T-cells are more } \\
\text { sensitive and specific to detect KFD from lupus lymphadenitis. Extensive necrosis seen on lymph node biopsy may suggest a possible } \\
\text { diagnosis of lupus lymphadenitis as the more likely diagnosis. }\end{array}$ \\
\hline 5 & $\begin{array}{l}\text { Systemic involvement of KFD with retroperitoneal lymphadenopathies may be associated with unusual presentations of flare-up (e.g., } \\
\text { lupus pancreatitis in our case) that require active, intensive and lengthy management. }\end{array}$ \\
\hline 6 & $\begin{array}{l}\text { Low serum complement levels }(\mathrm{C} 3, \mathrm{C} 4 \text { and } \mathrm{CH} 50) \text { in patients with } \mathrm{KFD} \text { potentially indicate a more severe, systemic and chronic } \\
\text { stage of the disease. These patients should be actively investigated and monitored for possible late onset of flare-ups. }\end{array}$ \\
\hline
\end{tabular}

ANA: antinuclear antibody; Anti-dsDNA: anti-double stranded DNA; APLA: antiphospholipid antibodies; KFD: Kikuchi-Fujimoto disease; RF, rheumatoid factor; SLE: systemic lupus erythematosus.

blood count with elevated LDH, although anemia of chronic disease (as seen in our case) is found in those with a more severe disease (12). Furthermore, presence of night sweats, nausea, vomiting, weight loss, diarrhea accompanying fever and lymphadenopathy (as in our patient), which represents the systemic form of $\mathrm{KFD}$, is more prominent in patients with extranodal involvement and could be associated with a more severe course of disease. This should trigger further assessment for aggressive forms of KFD, which tend to be fatal in some cases and only respond to early and intensive immunosuppressive therapy. Bearing in mind, making a diagnosis of KFD in patients with SLE might mislead clinicians to delay the possible diagnosis of lupus lymphadenitis, thereby delaying the appropriate treatment as may have been the case in our patient with enlarged retroperitoneal lymph nodes and the subsequent development of lupus pancreatitis.

\section{Conclusion}

Fortunately, even with systemic involvement, KFD is a self-limiting condition and it is imperative to recognize this condition in a patient who fits the epidemiological background, with typical clinical features, to avoid a burdensome investigative work up and adding unnecessary anxiety to the patient. Regular follow-up visits are particularly required in patients with KFD in a bid to detect possible SLE relapses/ onsets. Nevertheless, even without the occurrence of SLE, these patients should still be followed in proper intervals since recurrences of KFD itself can also continue to occur for many years (16).

\section{References}

1. Vijayaraghavan R, Chandrashekar R, Saraswathi A, Belagavi CS. Kikuchi-Fujimoto's disease involving mesenteric nodes:A report and review of literature. BMJ Case Rep. 2011; 2011. pii: bcr1020114945.

2. Kikuchi M. Lymphadenitis showing focal reticulum cells hyperplasia with nuclear debris and phagocytosis: A clinicopathological study. Nippon Ketsueki Gakkai Zasshi. 1972; 35:379-380.

3. Wilkinson CE, Nichol F. Kikuchi-Fujimoto disease associated with polymyositis. Rheumatology (Oxford). 2000; 39:1302-1304.

4. Ohta A, Matsumoto Y, Ohta T, Kaneoka H, Yamaguchi M. Still's disease associated with necrotizing lymphadenitis (Kikuchi's disease): Report of 3 cases. J Rheumatol. 1988; 15:981-983.

5. Imamura M, Ueno H, Matsuura A, Kamiya H, Suzuki T, Kikuchi K, Onoe T. An ultrastructural study of subacute necrotizing lymphadenitis. Am J Pathol. 1982; 107:292299.

6. Hollingsworth HC, Peiper SC, Weiss LM, Raffeld M, Jaffe ES. An investigation of the viral pathogenesis of Kikuchi-Fujimoto disease. Lack of evidence for EpsteinBarr virus or human herpesvirus type 6 as the causative agents. Arch Pathol Lab Med. 1994; 118:134-140.

7. Dorfman RF, Berry GJ. Kikuchi's histiocytic necrotizing lymphadenitis: An analysis of 108 cases with emphasis on differential diagnosis. Semin Diagn Pathol. 1988; 5:329-345.

8. Bosch X, Guilabert A, Miquel R, Campo E. Enigmatic Kikuchi-Fujimoto disease: A comprehensive review. Am J Clin Pathol. 2004; 122:141-152.

9. Kuo TT. Kikuchi's disease (histiocytic necrotizing lymphadenitis). A clinicopathologic study of 79 cases with an analysis of histologic subtypes, immunohistology, and DNA ploidy. Am J Surg Pathol. 
1995; 19:798-809.

10. el-Ramahi KM, Karrar A, Ali MA. Kikuchi disease and its association with systemic lupus erythematosus. Lupus. 1994; 3:409-411.

11. Zuo Y, Foshat M, Qian YW, Kelly B, Harper B, Karnath B. A rare case of Kikuchi Fujimoto's disease with subsequent development of systemic lupus erythematosus. Case Rep Rheumatol. 2012; 2012:325062.

12. Yu HL, Lee SS, Tsai HC, Huang CK, Chen YS, Lin HH, Wann SR, Liu YC, Tseng HH. Clinical manifestations of Kikuchi's disease in southern Taiwan. J Microbiol Immunol Infect. 2005; 38:35-40.

13. Kim SK, Kang MS, Yoon BY, Kim DY, Cho SK, Bae SC, Her MY. Histiocytic necrotizing lymphadenitis in the context of systemic lupus erythematosus (SLE): Is histiocytic necrotizing lymphadenitis in SLE associated with skin lesions? Lupus. 2011; 20:809-819.

14. Cramer J, Schmiedel S, Alegre NG, Schäfer H, Burchard GD, Merz H. Necrotizing lymphadenitis: KikuchiFujimoto disease alias lupus lymphadenitis? Lupus. 2010; 19:89-92.

15. Eisner MD, Amory J, Mullaney B, Tierney L Jr, Browner WS. Necrotizing lymphadenitis associated with systemic lupus erythematosus. Semin Arthritis Rheum. 1996; 26:477-482.

16. Litwin MD, Kirkham B, Henderson DR, Milazzo SC. Histiocytic necrotising lymphadenitis in systemic lupus erythematosus. Ann Rheum Dis. 1992; 51:805-807.

(Received July 8, 2016; Revised September 25, 2016; Accepted September 28, 2016) 